\title{
Danielle Constantin, Masques et Mirages. Genèse du roman chez Cortázar, Perec et Villemaire, 2008
}

\section{Claudia Amigo Pino}

\section{(2) OpenEdition}

$\checkmark$ Journals

\section{Édition électronique}

URL : http://journals.openedition.org/genesis/417

DOI : $10.4000 /$ genesis. 417

ISSN : 2268-1590

Éditeur :

Presses universitaires de Paris Sorbonne (PUPS), Société internationale de génétique artistique littéraire et scientifique (SIGALES)

Édition imprimée

Date de publication : 10 octobre 2010

Pagination : 168-170

ISBN : 978-2-84050-711-6

ISSN : 1167-5101

\section{Référence électronique}

Claudia Amigo Pino, «Danielle Constantin, Masques et Mirages. Genèse du roman chez Cortázar, Perec et Villemaire, 2008 », Genesis [En ligne], 31 | 2010, mis en ligne le 18 juin 2013, consulté le 22 septembre 2020. URL : http://journals.openedition.org/genesis/417 ; DOI : https://doi.org/10.4000/genesis.417 
On distinguera donc dans cet ouvrage les études fondées sur des archives matérielles, manuscrites et imprimées, de celles qui reposent sur des archives virtuelles, entendons sur l'absence d'archives. Les secondes revivifient, à la lumière de la génétique, les études de genèse qui figurent depuis Lanson au programme de l'histoire littéraire. Les premières, dans une démarche qui peut être authentiquement génétique, invitent à tenir compte de différences historiques cruciales : Érasme écrivait dans les ateliers d'imprimerie d'Alde Manuce et de Froben, La Bruyère dans celui de Michallet. Cela porte à conséquence.

Martine Sagaert, Peter Schnyder, André Gide. L'Écriture vive, Pessac, Presses universitaires de Bordeaux, coll. « Horizons génétiques », 2008, 168 p., avec DVD-Rom.

\section{Compte rendu par Catherine Viollet}

Premier maillon d'une collection prometteuse, intitulée « Horizons génétiques », aux Presses universitaires de Bordeaux, le volume André Gide. L'Écriture vive est à la fois séduisant et convaincant. Les deux spécialistes d'André Gide que sont Martine Sagaert (éditrice notamment du tome II du Journal, 1926-1950 dans la «Bibliothèque de la Pléiade ») et Peter Schnyder (éditeur d'une récente anthologie des Journaux de Maria van Rysselberghe, la « petite dame » proche de Gide, sous le titre Je ne sais si nous avons dit d'impérissables choses, Gallimard, coll. « Folio », 2006) proposent un parcours riche et original des manuscrits et de l'œuvre de Gide. Et ce, sous deux formes : à la fois sérieuse (le livre) et ludique (le DVD), qui se complètent à merveille, mais que l'on peut aussi bien apprécier indépendamment l'un de l'autre.

Commençons par le sérieux. Un volume à deux voix, d'environ cent soixante pages, qui regroupe plusieurs champs de recherche, et propose des sondages au plus près des manuscrits de l'écrivain :
- l'écriture du Journal, la mystérieuse genèse d'Ainsi soit-il, et la rédaction non moins complexe du Retour de l'URSS et de ses Retouches (Martine Sagaert) ;

- et un André Gide moins connu des lecteurs, correcteur d'épreuves et traducteur de Shakespeare ou de Friedrich Hebbel (Peter Schnyder) - analyses s'appuyant sur des documents inédits.

Le tout précédé d'une introduction, qui explique à quel point l'approche génétique sied particulièrement bien à l'auteur du Journal des Faux-Monnayeurs, plus intéressé par la fabrique du texte que par le texte lui-même, et fasciné par les manuscrits d'autres écrivains.

Après une présentation synthétique des différents modes d'édition de l'œuvre (critique ou génétique, par exemple pour Les Caves du Vatican), chacun des chapitres montre un aspect spécifique du travail de Gide - qu'il s'agisse des modalités pratiques de la tenue du Journal, des phases de rédaction de l'œuvre ultime Ainsi soit$i l$, ou de la dialectique des corrections apportées au récit du voyage en URSS. Les chapitres portant sur l'activité de Gide traducteur mettent en perspective les choix théoriques et pratiques de l'écrivain, et reproduit des documents inédits avec les variantes des traductions.

«Une œuvre qui déborde largement l'espace des livres publiés », affirment les auteurs. Et plus encore que cet ouvrage, c'est le DVD-Rom qui l'accompagne qui en fournit la preuve éclatante. Conçu de manière ludique, ce support propose plusieurs espaces où flâner à son gré :

- les manuscrits des autres (ceux auxquels Gide s'est particulièrement intéressé : Montaigne, Rilke et Charles-Louis Philippe);

- les documents présentant les travaux de Gide lui-même (soit quatre carnets du journal intégralement reproduits en facsimilé, les cent quarante-six feuillets correspondant à la rédaction d'Ainsi soit-il, des manuscrits inédits de la première traduction d'Hamlet ainsi que de celle des lettres de Hebbel sur la France);
- le « laboratoire de la création » porte sur les lieux, supports et instruments d'écriture, aspects matériels et concrets du processus scriptural, représentés sous forme de photos ou de témoignages de l'écrivain ;

- la section « L'œuvre à son estuaire » diffère et surprend par sa conception. Il s'agit d'une présentation commentée et vivante des « espaces papier du Journal », de l' « espace cahier » d'Ainsi soit-il et des modalités de sa rédaction, de lectures d'extraits ; et enfin de l'analyse détaillée, sur le motif, du dernier et énigmatique feuillet de cette œuvre, rédigé quelques jours avant la mort de l'écrivain - démonstration rigoureuse et fort concluante de ce que peut apporter l'analyse d'un document manuscrit.

Une vaste documentation bibliographique et iconographique s'ajoute à l'ensemble, sous le titre «médiathèque ». Chaque section est présentée par un commentaire clair et synthétique, guidant le parcours du lecteur. D'autres agréables surprises l'attendent, qui traduisent l'admiration que portent à Gide les deux chercheurs, et transmettent leur enthousiasme.

Danielle Constantin, Masques et Mirages. Genèse du roman chez Cortázar, Perec et Villemaire, New York, Peter Lang Publishing, 2008, 192 p.

\section{Compte rendu par Claudia Amigo Pino}

On a beau chercher au début du livre, on ne trouve pas de pistes expliquant les masques et mirages du titre. Ce n'est qu'au cours de la lecture qu'on découvre qu'ils renvoient à la figure de l'auteur, qui, dans les trois processus d'écriture analysés (Marelle, de Cortázar ; La Vie mode d' emploi, de Perec ; et La Vie en prose, de Yolande Villemaire) se cache, se cherche, se projette sur des personnages et des métaphores, et finalement se confond avec la figure du lecteur. 
Il s'agit bien d'un ouvrage de critique génétique, avec des descriptions du support, des reconstitutions du mouvement de l'écriture, et une enquête autour des discours sur la création de chacun des auteurs (entretiens, lettres, témoignages). Mais grâce à ce regard persistant sur les masques et mirages du scripteur, le texte ne se perd pas dans les détails et peut être lu avec autant d'enthousiasme que les romans étudiés.

Tout de même, cette étude n'a pu se faire sans une consultation exhaustive des fonds manuscrits des trois auteurs, qui se trouvent être très éloignés les uns des autres : celui de Cortázar est à la Benson Latin American Collection (BLAC, University of Texas à Austin), celui de Perec à l'Association Georges Perec (Bibliothèque de l'Arsenal, BnF, Paris) et celui de Yolande Villemaire à la collection d'archives privées de la Bibliothèque et des Archives nationales du Québec (BAnQ, Montréal). Après une description succincte du dossier génétique, chaque chapitre d'analyse présente des hypothèses générales sur le processus d'écriture, donnant lieu à une réflexion plus poussée sur la problématique de la mise en place de la figure auctoriale.

Ainsi, dans le cas de Marelle (Buenos Aires, 1963), on apprend que le dossier du roman n'est composé que d'un dactylogramme rédactionnel, deux feuillets et quelques chapitres complémentaires. À première vue, il s'agit d'un ensemble très lacunaire ; il est pourtant enrichi de la lecture du Cuaderno de Bitácora (« Journal de bord »), que Cortázar a tenu au cours de l'écriture du roman, publié par Ana María Barrenechea en 1983. Cette lecture permet d'affirmer, dès le départ, que Cortázar n'utilise pas de plans généraux préalables et travaille davantage par processus que par programme, selon la distinction proposée par Louis Hay et reprise par Almuth Grésillon. Quelques fragments du dactylogramme ont été écrits apparemment au fur et à mesure de la conception, dans un processus très semblable à l'écriture automatique proposée par le surréa- lisme. Mais Danielle Constantin souligne que « le fait que Rayuela soit un roman qui s'engage à fond dans des problèmes de métaphysique, de morale et d'esthétique peut difficilement présupposer une rédaction qui aurait résulté entièrement d'une écriture automatique, toujours aux confins de l'inconscient » (p. 61). En fait, dans le Journal de bord, on trouve des arrêts, des réflexions sur cette écriture en cours, des questionnements sur l'ordre, ce qui va déterminer des changements dans la structure comme dans l'écriture elle-même. C'est ce qui arrive à la figure de l'auteur, d'abord liée au personnage d'Horacio, qui occupe à la fois la place du héros, énonciateur, scripteur et auteur du texte, mais qui perd peu à peu une partie de ses fonctions. À son tour, le personnage de Morelli gagne de l'importance non pas en tant qu'auteur du texte, mais simplement en tant qu'auteur. Ce mouvement empêche le lecteur de relier très directement la figure de l'auteur à un personnage, et lui permet, en revanche, de prendre cette place d'énonciateur pour commencer à jouer à la marelle, comme le propose le roman.

Malgré les affinités esthétiques entre les romans de Cortázar et de Perec, en matière d'écriture, La Vie mode d'emploi (Paris, 1978) pourrait être définie comme l'exact contraire de Marelle. Au total, le processus de création du roman de Perec a duré une dizaine d'années, consacrées surtout à concevoir comment le livre serait écrit, comme on peut l'observer dans le Cahier des charges de La Vie mode d'emploi, publié en 1995 par Hans Hartje, Bernard Magné et Jacques Neefs. Danielle Constantin, pour sa part, a préféré se pencher sur l'entrée en rédaction en étudiant différents brouillons et dactylogrammes écrits entre 1975 et 1977. D'après Le Cahiers des charges, tout était préprogrammé : les personnages, les meubles, les vêtements, et même le nombre de pages de chaque chapitre. Le seul aspect du roman manquant au programme était l'intrigue principale, l'histoire de la vengeance de l'artisan Gaspard Winckler sur le millionnaire Percival Bartlebooth : cette histoire apparaîtra et sera développée au cours de la rédaction du roman, et déterminera un remaniement des éléments rassemblés jusqu'alors. L'analyse se concentre surtout sur les changements de l'emploi des pronoms sujets : au début, Perec préfère utiliser le « nous », une sorte de mirage du lecteur qui regarderait un texte impersonnel, qui serait là comme un immeuble est au milieu d'une rue. Mais peu à peu, ce « nous » est remplacé par un « je » utilisé par le personnage Valène, qui essaye de peindre une toile de tout ce qui est en train d'avoir lieu dans l'immeuble. Le lecteur ne serait plus confronté à un texte impersonnel, mais au discours d'un personnage qui prendrait pour soi la figure auctoriale. Ensuite, Perec change d'avis, retire ce « je » à Valène ; mais le récit reste focalisé sur ce personnage, décrit comme un passage de la voix au regard. Ainsi, «Valène, en ne faisant plus écran aux figures de l'auteur et du scripteur, alloue plus de liberté pour l'inscription multipliée de ces figures » (p. 117). On en vient donc à la même conclusion que Marelle : l'auteur d'abord se cache, puis se masque sous la figure d'un personnage, puis se multiplie dans plusieurs mirages, pour que le lecteur puisse jouer plus aisément au puzzle proposé par le roman.

Yolande Villemaire explore cette même procédure au cours de la rédaction de $L a$ Vie en prose (Montréal, 1980), mais « elle se dissimule astucieusement dans les recoins de son texte tout en multipliant jusqu'au vertige les usages du “je" » (p. 121). Tout comme Cortázar, l'écriture de Villemaire ne suit pas de programme, elle se montre plutôt « libre » et se déploie sur les différents supports du dossier (carnets, cahiers, calepins, feuilles volantes) sans ordre précis, hésitant entre la rédaction et la réflexion métascripturale ; elle parcourt diverses expérimentations linguistiques, surtout pour composer des anagrammes et leurs variations, hypogrammes, polygrammes et paragrammes (par exemple : la vie en prose/l'avis en prose/la vit en prose). Dès les premiers balbutiements du roman, il était question d'un « je », mais 
au cours de l'écriture, on ne sait plus de quel « je » il s'agit : l'analyse des brouillons rédactionnels dévoile un mouvement de multiplication de narratrices, de voix et de niveaux de récit, qui se répètent et se confondent comme dans des anagrammes narratifs. Au milieu de cette dissémination des sujets, un élément du récit reste débout : le « tu », qui apparaît dans tous les récits comme l'amoureux, l'objet désiré, le jumeau, bref, le lecteur, celui qui pourrait rassembler les récits. Mais tout comme chez Cortázar et chez Perec, on n'arrivera jamais au ciel de la marelle, le puzzle ne pourra pas être achevé, et le lecteur, bien sûr, se perdra comme les narratrices infinies de Villemaire.

Même si l'on y trouve des épilogues, même si les personnages sont morts, les écritures de ces trois romans postmodernes ne finissent pas, puisque la tâche de jouer, de rassembler, est à la charge du lecteur, qui ne peut pas mourir. Mais cette immortalité de l'écriture n'était pas donnée d'emblée. Comme l'a bien montré Danielle Constantin, l'auteur était bien vivant au début, dans les premières campagnes d'écriture : il a fallu passer par plusieurs miroitements entre carnets et brouillons, méta-écriture et rédaction, personnages et narrateurs et des tout petits bouts de phrase, pour que l'auteur soit bien mort et le lecteur plus vivant que jamais.

Modèles linguistiques, «Génétique de la production écrite et linguistique », coordonné par Irène Fenoglio et JeanMichel Adam, t. XXX, vol. LIX, 2009.

\section{Compte rendu par Louis Hay}

Le dernier numéro de Modèles linguistiques paraît sous le signe de l'ouverture.

Ouverture à une recherche internationale, à une diversité de corpus et à une pluralité d'écoles théoriques, qui apportent ensemble un sang neuf à la recherche. De là peut-être l'idée d'en proposer la lecture à un non-spécialiste, aux risques et périls de la linguistique.
Dans l'introduction du volume, JeanLouis Lebrave fait le point sur l'état d'une « linguistique de la production écrite » dont l'élaboration est l'un des acquis majeurs de la science génétique. Pour qui a suivi les recherches initiées par l'équipe de Linguistique de l'ITEM, les avancées sont sensibles : les concepts se sont affirmés, simplifiés et clarifiés. Désormais, le champ est nettement délimité : la linguistique ne saurait épuiser les singularités sémiotiques du manuscrit, ni saisir le processus même de l'écriture. Mais une théorie de l'énonciation écrite est possible qui permet de construire une génétique des transitions entre états. La voie est ouverte au travail de modélisation et Lebrave rappelle les quatre concepts qui décrivent classiquement les substitutions orientées dans le temps. Il propose par ailleurs de les compléter par une typologie chronologique de quatre étapes (cinq avec celle de la transmission) des processus d'écriture. Il faut citer sa conclusion qui ouvre, discrètement encore, une perspective qui porte loin : « En montrant ainsi comment le sens advient au texte dans les brouillons, la linguistique apporte une réponse originale à la question souvent débattue, de savoir si la critique génétique est ou non une critique. »

La question n'est pas étrangère au travail d'Irène Fenoglio qui figure un peu plus loin. On sait sa «découverte » du fonds Benveniste dans les tiroirs du département des Manuscrits orientaux de la BnF. L'analyse qu'elle en propose est à la fois une contribution à l'histoire de la recherche en sciences humaines et un exemple d'application d'une méthode génétique qui va du premier classement des manuscrits à l'analyse documentaire du dossier et à l'interprétation linguistique du texte. Le document étudié est l'essai sur « Le langage et l'expérience humaine », vaste réflexion sur la subjectivité et le temps où se développe notamment le concept fondamental de discours. Irène Fenoglio en fait l'objet d'une véritable leçon d'analyse microgénétique qui montre le surgissement de la pensée dans la langue, grâce à l'étude exhaustive d'une série complète d'échantillons repré- sentatifs des divers niveaux du manuscrit. De ce parcours, elle rapporte aussi des suggestions pour une réflexion, à peine ébauchée jusqu'ici, sur les traits distinctifs d'une écriture fictionnelle et d'une écriture scientifique.

Entre ces contributions figurent deux études consacrées aux rapports entre la linguistique de la genèse et celle du texte. Issues d'un programme interdisciplinaire de l'université de Lausanne, elles contribuent incontestablement à l'originalité de ce volume.

Jean-Michel Adam montre que la " transition entre états » relevée par Lebrave dans les manuscrits s'observe aussi bien dans les textes publiés. Il en présente une véritable typologie à travers une palette d'exemples convaincants du $\mathrm{XX}^{\mathrm{e}}$ siècle et propose, avec l'étude des trois états auctoriaux de La Belle au bois dormant de Perrault une démonstration qui fera chaud au cœur de tout généticien. Je suis un peu plus hésitant à le suivre jusqu'à la Chanson de Roland. Le statut de l'auteur, de l'œuvre, de la transmission me semble trop changé depuis l'époque médiévale pour s'intégrer à une démonstration contemporaine. Mais cela ne porte en rien préjudice à sa description du champ de la variation textuelle (p. 33) ni au schéma (p. 29) qui apporte un véritable changement de paradigme à la notion même de texte. Adam propose de lui substituer le concept englobant de textualité, qui place le texte au cœur d'un ensemble d'interactions qui le constituent : cotextes (d'un même recueil), métatextes (qui le glosent), interdiscursivité constituée par l'ensemble des systèmes de genres et de la langue disponibles à une époque donnée. Ce schéma justifie la double approche du texte par une linguistique de la genèse et une linguistique de la textualité. Il incite les généticiens à garder dans leur pensée une perspective ouverte sur le destin public des Euvres. Mais sans doute voudront-ils toujours distinguer entre les changements apportés au second Don Quichotte par Cervantès et ceux qu'il subirait sous la plume de Pierre Ménard. 